\title{
Tocolisis de mantención con progesterona micronizada oral para la prevención del parto prematuro después de parto prematuro frenado
}

\author{
Choudhary M, Suneja A, Vaid NB, Guleria K, Faridi MM. Maintenance tocolisis with oral \\ micronized progesterone for prevention of preterm birth after arrested preterm labor. Int $J$ \\ GynaecolObstet 2014. pii: S0020-7292(14)00181-7.doi: 10.1016/j.ijgo.2014.01.019.
}

Análisis crítico: Constanza Ralph T, Jorge A. Carvajal C, PhD.

Unidad de Medicina Materno-Fetal, División de Obstetricia y Ginecología. Escuela de Medicina. Facultad de Medicina. Pontificia Universidad Católica de Chile.

\section{RESUMEN (1)}

Objetivo: Evaluar la eficacia de la terapia de mantención con progesterona micronizada oral para la prolongación del embarazo en los casos de parto prematuro frenado. Métodos: Noventa mujeres entre 24-34 semanas de embarazo, únicos, con membranas intactas y trabajo de parto prematuro frenado, fueron asignados al azar a recibir progesterona micronizada oral $(n=45)$ o placebo $(n=45)$, diariamente hasta las 37 semanas o el parto. Los resultados fueron comparados mediante la prueba t de Student, $x^{2}$, prueba exacta de Fisher y $x^{2}$ en rangos. Resultados: Progesterona micronizada oral prolongó significativamente el tiempo de latencia $(33,29 \pm 22,16$ vs $23,07 \pm 15,42$ días; $p=0,013)$. Análisis de rangos reveló una diferencia significativa en el tiempo medio al parto entre los 2 grupos $(p=0,014)$. Hubo un número significativamente menor de partos prematuros ( $33 \%$ vs $58 \%, p=0,034)$ y de recién nacidos bajo peso al nacer (37\% vs $64 \%$; $\mathrm{p}=0,017)$ y significativamente mayor peso de nacimiento $(2,44 \pm 0,58$ vs $2,14 \pm 0,47 \mathrm{~kg} ; \mathrm{p}=0,009)$ en el grupo con progesterona. Los resultados perinatales y efectos adversos fueron similares en los 2 grupos. Conclusión: La tocolisis de mantención con progesterona micronizada oral significativamente prolongó el embarazo y disminuyó el número de partos prematuros.

\section{ANÁLISIS DE LA INVESTIGACIÓN}

\section{A. Relevancia de la investigación}

El parto prematuro, que corresponde al $10 \%$ del total de nacimientos a nivel mundial, es la primera causa de morbimortalidad neonatal (2). Su incidencia en Chile es cercano al 6\% (3). La morbimortalidad secundaria a la prematurez ha ido disminuyendo. Lamentablemente, esta reducción no se debe a una mejoría en las estrategias preventivas y terapéuticas de pacientes en riesgo o del manejo del parto prematuro, sino más bien al uso de corticoides y a un avance en el cuidado neonatal de los prematuros $(3,4)$.

La morbilidad neonatal de los prematuros depende de varios factores, pero principalmente del peso de nacimiento y de la edad gestacional al nacer (5). El manejo actual incluye uso de corticoides para inducir madurez pulmonar y tocolíticos, lo cual logra prolongar la gestación en 2-7 días, dando espacio para que actúen los corticoides y permitir, en algunos casos, el traslado materno con el fin de facilitar el ingreso del recién nacido a una unidad de cuidados intensivos (UCI) adecuada (6).

Existe discusión sobre el beneficio de la tocolisis de mantención luego de la frenación aguda en mujeres con trabajo de parto prematuro. Varias drogas han sido usadas como tocolisis de mantención 
( $\beta$-agonistas, bloqueadores de canales de calcio, sulfato de magnesio, atosiban), pero ninguna de ellas ha demostrado resultados consistentes en prolongar el embarazo, reducir la tasa de parto prematuro o mejorar los resultados neonatales (7).

La progesterona ha sido usada en la prevención del parto prematuro, de modo profiláctico, en mujeres con cuello corto o con antecedentes de parto prematuro. Un par de estudios recientes apoyan el uso de progesterona como tocolítico de mantención luego de la frenación aguda con otro fármaco $(8,9)$. Sin embargo, la evidencia es insuficiente para recomendar el uso progesterona como tocolítico de mantención en pacientes con trabajo de parto prematuro, justificando la necesidad de más estudios, de buena calidad, para demostrar la eficacia de progesterona micronizada vía oral para la prolongación del embarazo en los casos de parto prematuro frenado.

\section{B. El estudio (1)}

Diseño: Estudio randomizado prospectivo doble ciego conducido por el Departamento de Obstetricia y Ginecología y el Departamento de Pediatría del Colegio Universitario de Ciencias Médicas (UCMS) y el Hospital Guru Teg Bahadur en India. Realizado entre octubre 2010 y abril 2012. El cálculo del tamaño muestral se efectuó asumiendo 12 días de desviación estándar, basados en los datos obtenidos en otro estudio que evaluó tocolisis de mantención (9). Así se determinó que era necesario reclutar 42 pacientes por grupo para detectar 12 días de diferencia en tiempo ganado de embarazo, con un nivel de significación de $5 \%$ y poder estadístico de $80 \%$. El análisis se realizó según intención de tratar. Las mujeres fueron asignadas al azar a tratamiento o placebo, el estudio fue doble ciego. El grupo tratamiento recibió progesterona micronizada 200 mg vía oral y el grupo placebo capsulas similares con lactosa. El tratamiento se inició luego de 48 horas de que la paciente se encontrara asintomática y al alta se les entregó el sobre con las tabletas para una semana, con control semanal. Se verificó el uso del medicamento. El resultado primario fue la prolongación del embarazo. Los resultados secundarios fueron número de partos prematuros, peso de nacimiento, edad gestacional al parto, readmisión por parto prematuro, admisión neonatal a $\mathrm{UCl}$, estadía neonatal en $\mathrm{UCl}$, morbilidad neonatal, síndrome distrés respiratorio (SDR), sepsis, enterocolitis necrotizante, mortalidad neonatal precoz. Pacientes: Mujeres con embarazo único entre 24-34 semanas con diagnóstico de síntomas de parto prematuro fueron reclutadas luego de haber recibido tocolisis satisfactoria con Nifedipino según el protocolo del hospital. El diagnóstico de síntomas de parto prematuro fue hecho por la presencia de 4 contracciones cada 20 minutos u 8 contracciones cada 60 minutos asociado a cambios cervicales progresivos, dilatación cervical de más de $1 \mathrm{~cm}$ o más de $80 \%$ de borramiento cervical. Se consideró tocolisis satisfactoria si la paciente no presentaba contracciones por más de 12 horas. A todas las pacientes se les descartó presencia de infección por medio de exámenes de sangre, orina y cultivos cérvico-vaginales. Se excluyeron pacientes con embarazo múltiple, rotura prematura de membrana, historia de metrorragia, restricción de crecimiento fetal, distrés fetal, anomalía fetal congénita e historia previa de tocolisis. Resultados: Se lograron reclutar 90 mujeres, 45 en cada grupo. Se perdieron 2 participantes del grupo progesterona y 3 del grupo placebo; no se describen las causas del abandono. Los resultados fueron analizados por intención de tratar. La edad gestacional promedio al momento de la consulta fue de 31,9 semanas para el grupo progesterona y 32,4 semanas para el grupo placebo. El periodo de latencia promedio fue significativamente mayor para el grupo progesterona (33 días vs 23 días) $(p=0,013)$. La edad gestacional al parto no fue significativamente mayor para el grupo progesterona $(p=0,07)$. En el grupo progesterona la mayoría tuvo su parto a las 37 semanas $(62 \%)$ mientras que en el grupo placebo la mayoría fue entre las 34-36 semanas (38\%) $(p=0,01)$. La tasa de parto prematuro fue significativamente menor para el grupo progesterona (33\%) en comparación con el grupo placebo $(58 \%)(p=0,03)$. El promedio de peso al nacer fue mayor para el grupo progesterona $(2440 \mathrm{~g})$ en relación al placebo (2140 $g)(p=0,009)$. La mayoría de los neonatos en ambos grupos fueron adecuados para la edad gestacional. No hubo diferencias significativas en el puntaje de Apgar al nacer ni a los 5 minutos, admisión a UCI neonatal, estadía en UCI, SDR, sepsis o mortalidad neonatal. Hubo una muerte neonatal en cada grupo. En el grupo progesterona fue asociada a prematurez extrema con SDR y la del grupo placebo asociada a sepsis y hemorragia pulmonar. La mayoría de los pacientes no reportaron efectos adversos. Cuatro mujeres en cada grupo experimentaron cefalea, epigastralgia y acné. Los síntomas de parto prematuro recurrieron en 46 pacientes. Estas mujeres se mantuvieron con el mismo tratamiento y no necesitaron terapia distinta para su manejo. 


\section{Análisis crítico}

Estudio de buena calidad, nivel de evidencia tipo lb. El diseño del estudio es adecuado, la asignación de los pacientes fue aleatorizada, doble ciego, lo que reduce el riesgo de sesgo. En el diseño del estudio no se identifica sesgo de selección, de intervención ni de detección. Las poblaciones estudiadas son comparables y sólo diferentes en la intervención estudiada. El estudio fue diseñado para estudiar prolongación del embarazo, y en eso fue exitoso (en promedio se prologó 10 días más en el grupo progesterona comparado con el grupo placebo). El estudio detectó una reducción en la tasa de parto prematuro asociada al uso de progesterona, sin embargo, el estudio no fue diseñado para demostrar reducción en la incidencia de parto prematuro, de modo que no es posible una conclusión definitiva sobre este resultado. El uso de la progesterona no se asoció a una reducción significativa en la morbilidad perinatal.

\section{Conclusiones}

Estudio de excelente calidad metodológica, que demuestra prolongación del embarazo mediante el uso de tocolisis de mantención con progesterona luego de frenación aguda. Sin embargo, los resultados de mayor relevancia clínica (tasa de parto prematuro y resultado perinatal) son resultados secundarios en este estudio y no permiten conclusiones definitivas. El estudio es interesante y sus hallazgos promisorios, sin embargo, no suficientes para recomendar de modo rutinario el uso de progesterona en mujeres con amenaza de parto prematuro.

\section{REFERENCIAS}

1. Choudhary M, Suneja A, Vaid NB, Guleria K, Faridi MM. Maintenance tocolisis with oral micronized progesterone for prevention of preterm birth after arrested preterm labor. Int J Gynaecol Obstet 2014. pii: S00207292(14)00181-7.doi:10.1016/j.ijgo.2014.01.019. [Epubahead of print] PubMed PMID: 24807871.

2. Reducing Perinatal and Neonatal Mortality. Child Health Research Project Special Report. http://www. harpnet.org/doc/spec3.pdf. Published October 1999. Accessed April 6, 2012.

3. Carvajal J., Oyarzún E. Parto prematuro. Capítulo 11. Alto riesgo Obstétrico, Segunda Edición. Capítulo 11. Pg. 179-200.

4. Robert D, Dalziel S. Antenatal corticosteroid for accelerating fetal lung maturation for women at risk of preterm birth. Cochrane Database Syst Rev 2006;(3):CD004454

5. Fanaroff AA, Stoll BJ, Wright LL, Carlo WA, Ehrenkranz RA, et al. Am J Obstet Gynecol 2007;196:147.e1-147. e8

6. Ables AZ, Romero AM, Chauhan SP. Use of calcium channel antagonist for preterm labor. Obstet Gynecol Clin North Am 2005;32(3):519-25.

7. Dodd JM, Crowther CA, Dare MR, Middleton P. Oral betamimetics for maintenance therapy after threatened preterm labour. Cochrane Data base Syst Rev 2006;1:CD003927.

8. Sharami SH, Zahiri Z, Shakiba M, Milani F. Maintenance therapy by vaginal progesterone after threatened idiopathic preterm labor: a randomized placebo-controlled double-blind trial. Int J Fertil Steril 2010;4(2):45-50.

9. Arikan I, Barut A, Harma M, Harma IM. Effect of progesterone as a tocolytic and in maintenance therapy during preterm labor. Gynecol Obstet Invest 2011;72(4):269-73.

10. Borna S, Sahabi N. Progesterone for maintenance tocolytic therapy after threatened preterm labor: a randomized controlled trial. Aust N Z J Obstet Gynaecol 2008;48(1):58-63. 